\title{
Non-Classical computation of active-, apparent- and reactive powers and of the power- and distortion factors
}

\author{
Harald Richter
}

Clausthal University of Technology, Arnold-Sommerfeld-Str. 1, 38678 Clausthal, Germany

\begin{abstract}
In this paper, new mathematical formulas for the computation of the apparent power, the active power, and the reactive power are given, together with equations for the power factor and the distortion factor. These formulas are applicable for all four operational states, in which electric power networks can be. The given results remain valid also in the difficult case of nonlinear AC generators and loads, which exhibit non-sinusoidal line voltages and currents. The results are obtained by means of a Thevenin equivalent voltage and resistance of the power grid for every load. The advantages of the formulas are that they can directly use Fourier coefficients and that approximations of them do not depend on the equivalent resistance. Applications are seen in photovoltaic energy systems and in industry 4.0, where nonlinear AC generators and nonlinear user loads exist, respectively, as well as in general power systems.
\end{abstract}

Keywords: Apparent power, active power, reactive power, power factor, distortion factor

\section{Introduction}

An electric power system as described in [1], [2] can be in one of several operational states, and it can change between these states at any point in time. Here, it is suggested to consider four states for AC generators and loads, which are especially relevant for the transition to renewable energies. These states are: 1) linear generators that are coupled with linear loads, 2) linear generators connected to nonlinear loads, 3) nonlinear generators with linear loads, and 4) nonlinear generators with nonlinear loads. Renewable energies, Industry 4.0 and the Internet of Things forces the system to be in the state four, which is detrimental for the quality of the line voltage and its current.

\subsection{Linear generators and linear loads}

In this operational mode, linear generators are delivering electrical energy to linear consumer loads. 'Linear' means that the internal resistance of the Thevenin [3] equivalent of an AC generator or of a consumer load is either no or at most only a linear function of the voltage present at the device. This is the classic case of power networks, in which synchronous AC generators are engaged that are feeding-in pure sinusoidal voltages. Additionally, consumer loads are mainly of Ohmic nature, with only a small fraction of linear reactive resistivity. Although this is the best case for a power grid, it is already history in most public networks.

\subsection{Linear generators and nonlinear loads}

In this situation, linear generators are feeding-in AC power into a public power grid that distributes it to mostly nonlinear loads. In that state, the current $i=i(t)$ that flows through a consumer load $R_{v}$, is nonsinusoidal, although the Thevenin equivalent voltage $u_{0}=u_{0}(t)$ of the AC generators is fully sinusoidal.

\footnotetext{
* Manuscript received October 25, 2015; revised January 18, 2016.

Corresponding author. Tel.: +491741830141; E-mail address: hri@tu-clausthal.de.

doi: $10.12720 /$ sgce.5.1.56-71
} 
This happens despite the fact that $u_{0}(t)$ is embossed into $R_{v}$ by the low internal resistance of the power grid. The first reason for non-sinusoidal currents is that the value of $\mathrm{Rv}$ is not a constant, but varies over time due to the value of the applied voltage $u(t)$. As a result, the time course of the current $i=i(t)$ is modulated by $R_{v}$ and does not follow exactly the periodic increases and decreases of $u(t)$. By this modulation, harmonic oscillations show up in $i(t)$. Additionally to the modulation comes a second effect, which is created by switched power supplies or any other switching devices, which also results in harmonic oscillations. The reason for the second problem is that each switching process is always accompanied by a large amount of harmonics, otherwise switching cannot take place. Both effects together are detrimental for the quality of the network.

Examples of nonlinear consumer loads are analogous power supplies with transformers that are operated in magnetic saturation, semiconductor diodes and bridge rectifiers, switched power supplies, switched power inverters for motors and drives, general voltage inverters, and inverters with saturated inductors as energy storage. Line distortions can be quantified by the distortion factor THDF, as it will be explained later. Furthermore, as soon as harmonic oscillations show up, the standard AC calculus of electrical engineering in the Gaussian plane is no longer applicable, and several key factors of the energy system must be calculated otherwise, which are the apparent power, the active power, and the reactive power. These measures are defined also for the nonlinear cases by IEEE [4], [5] and other standardization bodies. Finally, with the increasing digitization of society, industry 4.0 and the Internet of things, more and more non-linear loads will be dislocated in households, companies and public administrations, thus deteriorating the line quality stronger and stronger.

\subsection{Nonlinear generators and linear loads}

In this operational state, nonlinear generators are feeding-in distorted sinusoidal voltages into the public power grid, while the consumer loads are assumed to be mainly linear. Nonlinear generators exist in photovoltaic installations, for example, in form of switched power converters that transform the directvoltages of the PV-modules into proper AC for the public grid. This conversion disseminates distorted electrical energy through the grid's distribution network, and its medium and high-voltage networks as well. Also in the super-grid, which operates at extremely-high direct-voltages, switched power converters are indispensable for transforming $\mathrm{AC}$ into $\mathrm{DC}$ and vice versa, which results in nonlinear power generation and distribution of electricity to countries or continents. In order to reduce harmonic distortions before inputting them to public power grids, filters are engaged. However, they are not that much effective, because the filter parameters are static, which means they do not adapt to the prevailing situation.

\subsection{Nonlinear generators and nonlinear loads}

In this case, nonlinear generators supply the power grid with non-sinusoidal voltages that are transmitted to nonlinear consumer loads. Therefore, already distorted line voltages are additionally distorted by the loads, thus amplifying the harmonic oscillations. This scenario is the worst cast, but it will dominate after the energy transition, because of the many expected photovoltaic installations. Since the renewable energies are currently growing tremendously, it should be an important goal for a successful transition to renewables to maintain the quality of the line voltages. Another circumstance, which has an unfavorable impact on the quality of the line voltages, is the volatility of the renewables. This problem has nothing to do with possible voltage fluctuations because of clouds or wind changes, but with the management of the power grid, which is performed by central instances. The central grid management has to switch back and forth between renewable and conventional power stations or block units to balance supply and demand. However, conventional power stations are injecting pure sinusoidal voltages by means of synchronous AC generators, while photovoltaic installations are doing the opposite. As a consequence, the quality of the line voltage is changing permanently, when switching-on or cuttingoff power stations from photovoltaic or from conventional sources. This means that public power grids will create and distribute more and more harmonic oscillations, that are varying from location to location 
and that change dynamically over time in a way, which will be not foreseeable by the customers.

\subsection{Mathematical preconditions for the new formulas}

As mathematical preconditions for the formulas given in this article, all voltage and current time courses are assumed to be periodic and bandwidth limited [6]. This is the case for all power grids. Additionally, all voltages and currents have to be periodically Fourier [7] transformed in order to calculate their spectra. Because of the first condition, the spectra are not Fourier integrals but Fourier sums, which have a finite number of sine/cosine oscillations. As a result, only a finite number of Fourier coefficients is present. Furthermore, it is required that the spectra have no DC component. This has consequences for the fields of applicability and means that only AC power grids, generators and consumer loads are addressed, but not DC supergrids. Finally, the formulas neglect the effects of subharmonics lower than $50 / 60 \mathrm{~Hz}$, which are created by beat frequencies in the grid, and the effects of interharmonics as well. Interharmonics are oscillations, which are not integer multiplies of the line frequency. Under these assumptions, the formulas will hold.

\section{State-of-the-Art}

\subsection{Standards}

According to [4], [5] there are five notions of electric power: active power P, reactive power Q, complex power $\underline{S}$, apparent power $\mathrm{S}$ and instantaneous power $\mathrm{p}(\mathrm{t})$. These powers are standardized as follows: the instantaneous power is according to Eq. (1), the active power P is defined by Eq. (2).

$$
\begin{aligned}
& p(t)=u(t) \cdot i(t) \\
& P=\overline{p(t)}=\frac{1}{T} \int_{0}^{T} p(t) d t
\end{aligned}
$$

The apparent power S is set as Eq. (3), and Q is computed due to Eq. (4). In Eq. (2), T denotes the period

$$
\begin{aligned}
& S=U_{e f f} \cdot I_{e f f} \\
& Q=\sqrt{S^{2}-P^{2}}
\end{aligned}
$$

of the fundamental oscillation of $u(t)$. The effective values of $u(t)$ and $i(t)$ are according to Eq. (5).

$$
U_{e f f}=\sqrt{\frac{1}{T} \cdot \int_{0}^{T} u^{2}(t) d t}, I_{e f f}=\sqrt{\frac{1}{T} \cdot \int_{0}^{T} i^{2}(t) d t}
$$

Furthermore, distortions are measured by the Total Harmonic Distortion Fundamental (THDF) as given in Eq. (6). Both, $u(t)$ and $i(t)$ can have an own THDF. In Eq. (6), $U_{\text {effl }}$ is the effective value of the fundamental oscillation $\mathrm{u}_{I}(\mathrm{t})$, while $\mathrm{U}_{\text {eff }}$ is the effective value of the total voltage $\mathrm{u}(\mathrm{t})$, the same holds for the current $\mathrm{i}(\mathrm{t})$ analogously.

$$
T H D F_{u(t)}=\frac{\sqrt{U_{e f f}^{2}-U_{e f f l}^{2}}}{U_{e f f 1}}, T H D F_{i(t)}=\frac{\sqrt{I_{e f f}^{2}-I_{e f f 1}^{2}}}{I_{e f f 1}}
$$

All definitions given above are valid for the four states energy systems can be. However, the complex power $\underline{S}$ is valid only in state 1 and not considered here. State 1 is well known. Finally, the power factor is the extension of the active factor for states $2-4$ and is standardized as Eq. (7). 


$$
\lambda=\frac{P}{S}
$$

For the non-classical computation, the IEEE/ISO standards of Eq. (1)-Eq. (7) are used throughout.

\subsection{Lemmas}

Furthermore, the subsequent lemmas are needed: Eq. (8)-Eq. (10) are orthogonal relations according to [8], [9] and Eq. (11)-Eq. (12) is a trigonometric equivalence due to [10].

$$
\begin{aligned}
& \int_{0}^{T} \sin (n \omega t) \sin (m \omega t) d t=\left[\begin{array}{c}
0, \text { for } n \neq m \\
0, \text { for } n=m=0 \\
\frac{T}{2}, \text { for } n=m \neq 0
\end{array}\right] \\
& \int_{0}^{T} \cos (n \omega t) \cos (m \omega t) d t=\left[\begin{array}{c}
0, \text { for } n \neq m \\
T, \text { for } n=m=0 \\
\frac{T}{2}, \text { for } n=m \neq 0
\end{array}\right] \\
& \int_{0}^{T} \sin (n \omega t) \cos (m \omega t) d t=0, \text { for all }(n, m) \\
& \sin (\alpha \pm \beta)=(\sin \alpha \cos \beta \pm \cos \alpha \sin \beta) \\
& \cos (\alpha \pm \beta)=(\cos \alpha \cos \beta \mp \sin \alpha \sin \beta)
\end{aligned}
$$

With Eq. (11)-Eq. (12), the orthogonal relations can be expanded to phase-shifted sine and cosine (Eq. (13)-Eq. (15)) with any $\varphi_{1}, \varphi_{2} \in \mathbf{R}$.

$$
\begin{array}{r}
\int_{0}^{T} \sin \left(n \omega t+\varphi_{1}\right) \sin \left(m \omega t+\varphi_{2}\right) d t=\cos \varphi_{1} \cos \varphi_{2}\left[\begin{array}{c}
0, \text { for } n \neq m \\
0, \text { for } n=m=0 \\
\frac{T}{2}, \text { for } n=m \neq 0
\end{array}\right]+\sin \varphi_{1} \sin \varphi_{2}\left[\begin{array}{c}
0, \text { for } n \neq m \\
T, \text { for } n=m=0 \\
\frac{T}{2}, \text { for } n=m \neq 0
\end{array}\right] \\
\int_{0}^{T} \cos \left(n \omega t+\varphi_{1}\right) \cos \left(m \omega t+\varphi_{2}\right) d t=\cos \varphi_{1} \cos \varphi_{2}\left[\begin{array}{c}
0, \text { for } n \neq m \\
T, \text { for } n=m=0 \\
\frac{T}{2}, \text { for } n=m \neq 0
\end{array}\right]+\sin \varphi_{1} \sin \varphi_{2}\left[\begin{array}{c}
0, \text { for } n \neq m \\
0, \text { for } n=m=0 \\
\frac{T}{2}, \text { for } n=m \neq 0
\end{array}\right] \\
\int_{0}^{T} \sin \left(n \omega t+\varphi_{1}\right) \cos \left(m \omega t+\varphi_{2}\right) d t=\cos \varphi_{1} \sin \varphi_{2}\left[\begin{array}{c}
0, \text { for } n \neq m \\
0, \text { for } n=m=0 \\
\frac{T}{2}, \text { for } n=m \neq 0
\end{array}\right]+\sin \varphi_{1} \cos \varphi_{2}\left[\begin{array}{c}
0, \text { for } n \neq m \\
T, \text { for } n=m=0 \\
\frac{T}{2}, \text { for } n=m \neq 0
\end{array}\right](15)
\end{array}
$$

\section{New Computation Method for All States}

The new method is based on the Thevenin equivalent circuit given in Fig. 1, which is a model for the 
power grid as it it seen by any given user load $\mathrm{R}$. This means that the whole power grid that surrounds $\mathrm{R}$ is subsumed into one Thevenin equivalent circuit. The values of $\mathrm{u}_{l}(\mathrm{t})$ and $\mathrm{R}_{l}$ are determined by the position of $\mathrm{R}$ in the power system. For every load $\mathrm{R}$, a specific pair $\left(\mathrm{u}_{l}(\mathrm{t}), \mathrm{R}_{l}\right)$ is needed as model.

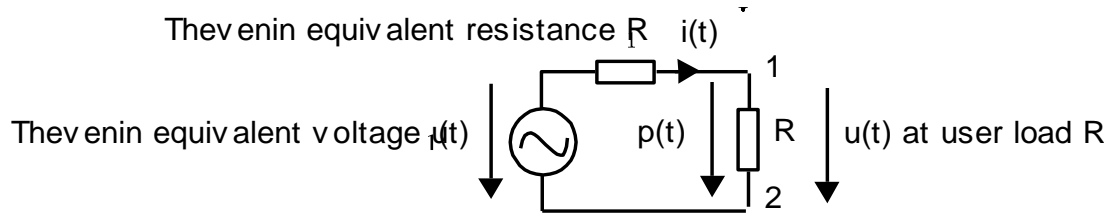

Fig. 1. Model of the power grid for load R. $\mathrm{u}_{l}(\mathrm{t})$ is the Thevenin equivalent voltage of the power grid, and $\mathrm{R}_{l}$ is its internal resistance.

\subsection{Thevenin equivalent voltage}

The time course of the Thevenin equivalent voltage is denoted as $\mathrm{u}_{l}(\mathrm{t})$. It can be measured by disconnecting the load $\mathrm{R}$ from the power grid and by sampling between the remaining terminals $(1,2)$ for a period $\mathrm{T}$ with a sampling frequency that matches the Nyquist theorem. Afterwards, the spectrum of $\mathrm{u}_{I}(\mathrm{t})$ can be calculated by means of the Fourier coefficients $\left(\hat{\mathrm{u}}_{n}, \alpha_{n}\right)$ for all $\mathrm{n}$. Furthermore, nonlinear AC generators in the power grid are modelled such that $\mathrm{u}_{I}(\mathrm{t})$ has harmonic oscillations, which are a Fourier sum that consists of $\left(\hat{\mathrm{u}}_{n}, \boldsymbol{\alpha}_{n}\right)$ as Fourier coefficients.

\subsection{Thevenin equivalent resistance}

The resistor $\mathrm{R}_{l}$ of the equivalent circuit in Fig. 1 is assumed to be a constant. It can be measured in the power grid in the moment of the zero-crossing of $\mathrm{u}_{l}(\mathrm{t})$ by means of a high-speed measurement. Furthermore, $\mathrm{R}$ is assumed to be mainly of ohmic nature, with no or only little inductive or capacitive fraction. As a result, the power in $\mathrm{R}_{l}$ is active power only, and $\mathrm{u}_{l}(\mathrm{t})-\mathrm{u}(\mathrm{t})$ and $\mathrm{i}(\mathrm{t})$, i.e. the voltage and the current over $\mathrm{R}_{l}$ are assumed to be in phase.

\subsection{Load R}

The load $\mathrm{R}$ is either a constant, if the power grid is in state is 1 or 3 , or it is a nonlinear function of $\mathrm{i}(\mathrm{t})$, if the state is 2 or 4 . A nonlinear load is modelled by an $\mathrm{i}(\mathrm{t})$ that has harmonic oscillations, which are a Fourier sum. Furthermore, $R$ can create a phase shift between $u(t)$ and $i(t)$. Therefore, all kinds of powers can exist at $\mathrm{R}$.

\subsection{Mathematical model for every state}

For every state of the power grid, a specific model is made for $\mathrm{u}_{I}(\mathrm{t})$ and $\mathrm{i}(\mathrm{t})$ in the following way:

1) For state 1 (lin. gen.+lin. load), Eq. (16) is used, which is the classical approach.

$$
u_{1}(t)=\hat{u}_{1} \sin (\omega t), i(t)=\hat{i}_{1} \sin (\omega t+\beta), R=\text { const }
$$

2) For state 2 (lin. gen.+nonlin. load), Eq. (17) is taken, which uses a Fourier sum for i(t).

$$
u_{1}(t)=\hat{u}_{1} \sin (\omega t), i(t)=\sum_{n=1}^{N} \hat{i}_{n} \sin \left(n \omega t+\beta_{n}\right), R=R(i(t))
$$

3) The state 3 (nonlin.gen.+lin. load) is described by Eq. (16), using Fourier sums for both $\mathrm{u}_{l}(\mathrm{t})$ and $\mathrm{i}(\mathrm{t})$.

$$
u_{I}(t)=\sum_{n=1}^{N} \hat{u}_{n} \sin \left(n \omega t+\alpha_{n}\right), i(t)=\sum_{n=1}^{N} \hat{i}_{n} \sin \left(n \omega t+\beta_{n}\right), R=\text { const }, N \in N
$$


4) For state 4 (nonlin. gen.+nonlin. load) Eq. (17), is used as model, which has also two Fourier sums,

$$
u_{l}(t)=\sum_{n=1}^{N} \hat{u}_{n} \sin \left(n \omega t+\alpha_{n}\right), i(t)=\sum_{n=1}^{M} \hat{i}_{n} \sin \left(n \omega t+\beta_{n}\right), R=R(i(t)), M \geq N \in N
$$

but the amount of harmonics at the load $\mathrm{R}$ is higher than in case 3, because of the modulation and/or switching of the already distorted input voltage $\mathrm{u}_{l}(\mathrm{t})$ by $\mathrm{R}$. In all four mathematical models, $\omega$ is calculated by $\omega=2 \pi \mathrm{f}=2 \pi(1 / \mathrm{T})$, with $\mathrm{f}$ as line frequency and $\mathrm{T}$ as line period. Furthermore, $\left(\hat{\mathrm{u}}_{n}, \alpha_{n}\right),\left(\hat{\mathrm{i}}_{n}\right.$, $\beta_{n}$ ) are the Fourier coefficients of the $n$-th harmonic of the equivalent voltage and of the current through

$\mathrm{R}$, respectively. For the value domain of the variables and constant $\left(\mathrm{t}, \omega, \mathrm{f}, \hat{\mathrm{u}}_{n}, \hat{\mathrm{i}}_{n}, \boldsymbol{\alpha}_{n}, \beta_{n}\right) \in \mathbf{R}$ holds. Additionally, the constants $\mathrm{N}, \mathrm{M}$ define the highest harmonic oscillations in the spectra of $\mathrm{u}_{l}(\mathrm{t})$ and $\mathrm{i}(\mathrm{t})$, respectively. Both are finite, due to the preconditions made.

\subsection{Method}

With that prerequisites and restrictions, the following method is suggested to be repeated in all states 2-4 of the energy system for an arbitrary amount of time:

1) Measure the time course of $\mathrm{u}_{l}(\mathrm{t})$ at the terminals $(1,2)$ while disconnecting $\mathrm{R}$ for one period $\mathrm{T}$ from zero crossing to zero crossing to avoid self-made switching distortions. This can be accomplished via an IGBT switch, for example. The number of the samples must match the Nyquist theorem.

2) Compute the coefficients $\left(\hat{\mathrm{u}}_{n}, \mathrm{\alpha}_{n}\right)$ of the Fourier sum of $\mathrm{u}_{I}(\mathrm{t})$ by means of a Fast Fourier Transform (FFT)

3) Measure the value of $\mathrm{R}_{I}$ in the moment, when $\mathrm{u}_{I}(\mathrm{t})$ has the zero crossing in the interval $\mathrm{T}$ of 1 ) by means of a high-speed measurement equipment for ohmic resistance. The detection of the zero crossing must be made quickly and with high-precision.

4) Measure the time course of $i(t)$ that flows through the load $R$ via a hall sensor in a clamp-on ammeter, for example, during the following time interval $\mathrm{T}$. The number of the samples must match the Nyquist theorem.

5) Compute the coefficients $\left(\hat{\mathbf{1}}_{n}, \beta_{n}\right)$ of the Fourier sum of $\mathrm{i}(\mathrm{t})$ by means of a FFT.

6) Compute $\mathrm{P}, \mathrm{S}, \mathrm{Q}, \lambda$, and THDF in the same interval by the formulas given subsequently

7) Repeat all steps 1-5 periodically with time interval $T_{2}>>2 T$, such that the active power through the load $\mathrm{R}$ is only little impaired by the disconnection of the load

The disadvantage of the proposed method is that the spectra of $\mathrm{u}_{l}(\mathrm{t})$ and $\mathrm{i}(\mathrm{t})$ cannot be obtained in the same period $\mathrm{T}$. Its first advantage is that the load $\mathrm{R}$ can be of any kind, including phase-shifting and nonlinear, because no formula depends on $\mathrm{R}$. The second advantage of the method is all of the subsequent formulas have exact and approximate solutions. The latter do not even depend on $\mathrm{R} 1$, which means that the measurement of the internal resistance of the power becomes obsolete for the approximations. The third advantage is that all key parameters of the power grid can be directly obtained from the Fourier coefficients of $\mathrm{u}_{l}(\mathrm{t})$ and $\mathrm{i}(\mathrm{t})$ by an FFT. An application for the given method is as follows: the operator of a photovoltaic power plant measures the Fourier coefficients of $\mathrm{u}_{l}(\mathrm{t})$ and $\mathrm{i}(\mathrm{t})$ according to the given method and computes the parameters $\mathrm{Q}, \lambda$ and THDF, while a consumer or a company are doing the same at their sides. All parties are sending these parameters to a central control room of the grid operator, who initiates a change in the filter settings of all stake holders to improve the quality of voltage and current in the energy system.

\section{Results for State 1 (lin. gen. + lin. loads)}

This state is not considered here, because all results are well known. 


\section{Results for State 2 (lin. gen. + nonlin. loads)}

\subsection{Active power $P$}

For the computation of $\mathrm{P}$, the voltage that drops over load $\mathrm{R}_{l}$ is according to Fig. 1 and calculated by Eq. (20) due to Kirchhoff's mesh rule.

$$
u(t)=u_{1}(t)-i(t) R_{1}
$$

Furthermore, the instantaneous power p(t) translates into (Eq. 21), because of Eq. (1) and Eq. (20).

$$
p(t)=u_{l}(t) \cdot i(t)=a+b
$$

The terms 'a' and 'b' are given in Eq. (22) and Eq. (23), respectively because of Eq. (17).

$$
\begin{aligned}
& a=\hat{u}_{I} \sum_{k=1}^{N} \hat{i}_{n} \sin \omega t \cdot \sin \left(n \omega t+\beta_{n}\right) \\
& b=-R_{I}\left\{\sum_{n=1}^{N} \hat{i}_{n} \sin n \omega t+\beta_{n}\right\}
\end{aligned}
$$

The computation of $\mathrm{P}$ requires the integration of Eq. (21). For that, Eq. (22) can be integrated first, which results in Eq. (24) by swapping integration with summation. This is possible, because both are linear operators. For computing the integral in Eq. (24), the lemma of Eq. (13) is applied with $\mathrm{n}=1, \varphi_{l}=0$ and $\varphi_{2}=\beta_{n}$. Because of that, $\cos \varphi_{1}=1$ and $\sin \varphi_{1}=0$ holds. As a result, the lemma of Eq. (13) removes all $\sin / \sin$ terms with different frequencies $n \omega t, m \omega t$, and only the term with $n=m=1$ remains, which is $(\mathrm{T} / 2) \hat{\mathrm{u}}_{1} \hat{\mathrm{l}}_{l} \cos \beta_{l}$.

$$
\int_{0}^{T} a d t=\hat{u}_{1} \sum_{n=1}^{N}\left\{\int_{0}^{T} \hat{i}_{n} \sin \omega t \cdot \sin \left(n \omega t+\beta_{n}\right) d t\right\}=\frac{T}{2} \cdot \hat{u}_{1} \hat{i}_{1} \cos \beta_{1}
$$

Then, Eq. (23) can be expanded to Eq. (25) by replacing the squared sum by a double sum.

$$
b=-R_{l}\left(\sum_{k=1}^{N} e_{k}\right)^{2}=-R_{l} \sum_{j=1}^{N} \sum_{k}^{N} e_{j} e_{k}, \text { with } e_{k}=\hat{i}_{k} \sin \left(k \omega t+\beta_{k}\right), e_{j}=\hat{i}_{j} \sin \left(j \omega t+\beta_{j}\right)
$$

The integration of the double sum of Eq. (25) results in Eq. (26), again by exchanging integration with summation.

$$
\int_{0}^{T} b d t=-R_{I} \sum_{k=1}^{N} \sum_{j=1}^{N}\left\{\int_{0}^{T}\left(e_{j} e_{k}\right) d t\right\}
$$

In Eq. (26), only the elements of the main diagonal $\mathrm{e}_{j} \mathrm{e}_{k=j}$ contribute to the integral. The integration over $\mathrm{e}_{j} \mathrm{e}_{k}$ with $\mathrm{k} \neq \mathrm{j}$ is zero because of Eq. (13). With Eq. (13), the result of the integral of Eq. (26) is obtained in Eq. (27).

$$
\int_{0}^{T}\left(e_{j} e_{j}\right) d t=\int_{0}^{T} \hat{i}_{j} \hat{i}_{j} \sin \left(j \omega t+\beta_{j}\right) \sin \left(j \omega t+\beta_{j}\right) d t=\frac{T}{2} \hat{i}_{j}^{2}\left(\cos ^{2} \beta_{j}+\sin ^{2} \beta_{j}\right)=\frac{T}{2} \hat{i}_{j}^{2}
$$

With Eq. (27), the result of the integration of b is given by Eq. (28). 


$$
\int_{0}^{T} b d t=-R_{1} \frac{T}{2} \cdot \sum_{k=1}^{N} \hat{i}_{k}^{2}
$$

Out of Eq. (2), Eq. (24) and Eq. (28), we obtain the final result for the active power P according to Eq. (29).

$$
P=\frac{1}{2} \cdot\left(\hat{u}_{1} \hat{i}_{1} \cos \beta_{l}-R_{1} \sum_{k=1}^{N} \hat{i}_{k}^{2}\right)
$$

This means that the active power at a nonlinear load $\mathrm{R}$ is defined by the effective value of the Thevenin equivalent voltage $\mathrm{u}_{l}(\mathrm{t})$ of the power grid and by the effective value of the fundamental oscillation of the current $\mathrm{i}(\mathrm{t})$ that flows through that load, subtracted by the active power that is created by $\mathrm{i}(\mathrm{t})$ and consumed by the Thevenin equivalent resistance. How much active power is transferred from the grid to the load is parametrized by the phase shift angle between $\mathrm{u}_{l}(\mathrm{t})$ and the fundamental oscillation of $\mathrm{i}(\mathrm{t})$. The result can be approximated for small $\mathrm{R}_{l}$ to Eq. (30), which is a known formula from state 1 of energy systems.

$$
P \approx \frac{u_{1} l_{l}}{2} \cdot \cos \beta_{1}
$$

Please note that $\mathrm{R}_{I}$ is small only for public power grids, because of the many AC generators that are available there. However, it is not small for a photovoltaic installation on the own roof, for example, that is operated as power source in island mode.

\subsection{Apparent power $S$}

The apparent power S must be computed via Eq. (3), Eq. (5) and Eq. (17). Again, Eq. (20) is used as starting point, as it was already the case for the calculation of P. Additionally, the computation of $\mathrm{I}_{\text {eff }}$ is performed first. It requires the squaring of $\mathrm{i}(\mathrm{t})$ and an subsequent integration according to Eq. (31).

$$
\int_{0}^{T} i^{2}(t) d t=\int_{0}^{T}\left\{\sum_{n=1}^{N} \hat{i}_{n} \sin \left(n \omega t+\beta_{n}\right)\right\}^{2} d t
$$

However, this operation was already performed for a similar case by Eq. (23) and Eq. (26), and the result can be taken from there, which means from Eq. (28). Consequently, the result is given by Eq. (32).

$$
\int_{0}^{T} i^{2}(t) d t=\frac{T}{2} \cdot \sum_{k=1}^{N} \hat{i}_{k}^{2}
$$

With that result, $\mathrm{I}_{\text {eff }}$ becomes as Eq. (33).

$$
I_{e f f}=\frac{1}{\sqrt{2}} \sqrt{\sum_{k=1}^{N} \hat{i}_{k}^{2}}
$$

Now, $\mathrm{U}_{\text {eff }}$ is calculated. It requires the squaring of $\mathrm{u}(\mathrm{t})$ according to Eq. (34).

$$
u^{2}(t)=\left[u_{1}(t)-i(t) R_{1}\right]^{2}=u_{1}^{2}-2 u_{1} i R_{1}+R_{1}^{2} i^{2}
$$

Subsequently, all three terms $\mathrm{u}_{l}{ }^{2},-2 \mathrm{u}_{l} \mathrm{i} \mathrm{R}_{l}$ and $\mathrm{R}_{l}{ }^{2} \mathrm{i}^{2}$ must be integrated. The integration of the first term is given by Eq. (35), and the result is obtained by applying Eq. (8) on it.

$$
\int_{0}^{T} u_{1}^{2}(t) d t=\int_{0}^{T} \hat{u}_{1}^{2} \sin ^{2} \omega t d t=\hat{u}_{1}^{2} \frac{T}{2}
$$


The integration of the middle term is given by Eq. (36) by means of Eq. (17).

$$
\int_{0}^{T}\left(-2 u_{1} i R_{l}\right) d t=-2 R_{1} \hat{u}_{1} \int_{0}^{T}\left(\sum_{n=1}^{N} \hat{i}_{n} \sin \omega t \cdot \sin \left(n \omega t+\beta_{n}\right)\right) d t=-T R_{1} \hat{u}_{1} \hat{i}_{1} \cos \beta_{1}
$$

The result is obtained by swapping integration and summation and by applying Eq. (18) as it was already shown in Eq. (24). The integration of the last term can be obtained via Eq. (32) and results in Eq. (37).

$$
\int_{0}^{T} R_{l}^{2} i^{2}(t) d t=\frac{T}{2} R_{l}^{2} \cdot \sum_{k=1}^{N} \hat{i}_{k}^{2}
$$

Out of Eq. (37), Eq. (36) and Eq. (35), the integral over the squared u(t) can be composed as Eq. (38).

$$
\int_{0}^{T} u^{2}(t) d t=\hat{u}_{1}^{2} \frac{T}{2}-T R_{l} \hat{u}_{1} \hat{i}_{l} \cos \beta_{l}+\frac{T}{2} R_{I}^{2} \cdot \sum_{k=1}^{N} \hat{i}_{k}^{2}
$$

With Eq. (5) and Eq. (38), $\mathrm{U}_{\text {eff }}$ becomes Eq. (39). From Eq. (33) and Eq. (39), the final result for $\mathrm{S}$ is Eq. (41).

$$
\begin{aligned}
& U_{\text {eff }}=\frac{1}{\sqrt{2}} \sqrt{\hat{u}_{l}^{2}-2 R_{l} \hat{u}_{l} \hat{i}_{1} \cos \beta_{l}+R_{l}^{2} \cdot \sum_{k=1}^{N} \hat{i}_{k}^{2}} \\
& S=\frac{1}{2} \sqrt{\hat{u}_{l}^{2}-2 R_{l} \hat{u}_{l} \hat{i}_{l} \cos \beta_{1}+R_{l}^{2} \cdot \sum_{k=1}^{N} \hat{i}_{k}^{2}} \cdot \sqrt{\sum_{k=1}^{N} \hat{i}_{k}^{2}}
\end{aligned}
$$

For small $\mathrm{R}_{l}$ holds approximately Eq. (41).

$$
S \approx \frac{\hat{u}_{1}}{2} \sqrt{\sum_{k=1}^{N} \hat{i}_{k}^{2}}
$$

\subsection{Reactive power $Q$}

The reactive power Q is computed out of Eq. (29) and Eq. (40) by means of Eq. (4), yielding Eq. (42).

$$
Q^{2}=\left(\frac{1}{4} \cdot\left(\hat{u}_{1}^{2}-2 R_{1} \hat{u}_{1} \hat{i}_{1} \cos \beta_{1}+R_{1}^{2} \cdot \sum_{k=1}^{N} \hat{i}_{k}^{2}\right) \sum_{k=1}^{N} \hat{i}_{k}^{2}\right)-\frac{1}{4} \cdot\left(\hat{u}_{1} \hat{i}_{1} \cos \beta_{1}-R_{1} \sum_{k=1}^{N} \hat{i}_{k}^{2}\right)^{2}
$$

The final result is Eq. (43).

$$
Q=\frac{1}{2} \sqrt{\left(\hat{u}_{l}^{2}-2 R_{1} \hat{u}_{1} \hat{i}_{l} \cos \beta_{1}+R_{l}^{2} \cdot \sum_{k=1}^{N} \hat{i}_{k}^{2}\right)\left(\sum_{k=1}^{N} \hat{i}_{k}^{2}\right)-\left(\hat{u}_{1} \hat{i}_{1} \cos \beta_{l}-R_{l} \sum_{k=1}^{N} \hat{i}_{k}^{2}\right)^{2}}
$$

This reduces to Eq. (44) for small $\mathrm{R}_{l}$.

$$
Q \approx \frac{\hat{u}_{1}}{2} \sqrt{\left(\sum_{k=1}^{N} \hat{i}_{k}^{2}\right)-\hat{i}_{1}^{2} \cos ^{2} \beta_{1}}
$$

\subsection{Power factor $\lambda$}

The power factor $\lambda$ is computed out of Eq. (29) and Eq. (40) by means of Eq. (7), yielding Eq. (46). 


$$
\lambda=\frac{\left(\hat{u}_{1} \hat{i}_{1} \cos \beta_{I}-R_{I} \sum_{k=1}^{N} \hat{i}_{k}^{2}\right)}{\sqrt{\hat{u}_{l}^{2}-2 R_{l} \hat{u}_{l} \hat{i}_{l} \cos \beta_{I}+R_{I}^{2} \cdot \sum_{k=1}^{N} \hat{i}_{k}^{2}} \cdot \sqrt{\sum_{k=1}^{N} \hat{i}_{k}^{2}}}
$$

This reduces to Eq. (46) for small $\mathrm{R}_{l}$.

$$
\lambda \approx \frac{\hat{i}_{1} \cos \beta_{1}}{\sqrt{\sum_{k=1}^{N} \hat{i}_{k}^{2}}}
$$

\subsection{THDF for voltage and current at load $R$}

First, the THDF for the voltage $\mathrm{u}(\mathrm{t})$ at load $\mathrm{R}$ is computed according to its definition in Eq. (6) and by means of Eq. (39), yielding Eq. (47).

$$
T H D F_{u}=\sqrt{\frac{R_{l}^{2} \cdot \sum_{k=2}^{N} \hat{i}_{k}^{2}}{\hat{u}_{l}^{2}-2 R_{l} \hat{u}_{l} \hat{i}_{l} \cos \beta_{l}+R_{l}^{2} \cdot \hat{i}_{l}^{2}}}
$$

This reduces to zero for small $\mathrm{R}_{l}$. The computation of the THDF for the current $\mathrm{i}(\mathrm{t})$ can be performed via Eq. (33), resulting in Eq. (48), which is already a simple expression.

$$
\operatorname{THDF}_{i}=\frac{\sqrt{\sum_{k=2}^{N} \hat{i}_{k}^{2}}}{\hat{i}_{1}}
$$

\section{Results for State 3 (nonlin. gen. + lin. loads)}

For this state, the Thevenin equivalent voltage $\mathrm{u}_{l}(\mathrm{t})$ becomes a Fourier sum as given in Eq. (18). Every harmonic of $\mathrm{u}_{1}(\mathrm{t})$ creates a harmonic oscillation in $\mathrm{i}(\mathrm{t})$ that can additionally be phase-shifted. However, no new harmonics come into existence, because of the linearity of $\mathrm{R}$.

\subsection{Active power $P$}

For the computation of $\mathrm{P}$, the starting point is again Kirchhoff‘s mesh rule, as given in Eq. (20).

$$
u(t)=u_{1}(t)-i(t) R_{1}=\sum_{n=1}^{N} \hat{u}_{n} \sin \left(n \omega t+\alpha_{n}\right)-R_{1} \sum_{n=1}^{N} \hat{i}_{n} \sin \left(n \omega t+\beta_{n}\right)
$$

The instantaneous power $\mathrm{p}(\mathrm{t})$ translates into Eq. (50).

$$
p(t)=u_{l}(t) \cdot i(t)=c+f
$$

The terms ' $c$ ' and ' $f$ ' are given in Eq. (51) and Eq. (52), respectively.

$$
\begin{aligned}
& c=\left(\sum_{j=1}^{N} \hat{u}_{j} \sin \left(j \omega t+\alpha_{j}\right)\right) \cdot \sum_{k=1}^{N} \hat{i}_{k} \sin \left(k \omega t+\beta_{k}\right) \\
& f=-R_{l}\left\{\sum_{n=1}^{N} \hat{i}_{n} \sin n \omega t+\beta_{n}\right\}
\end{aligned}
$$


The computation of $\mathrm{P}$ requires the integration of ' $\mathrm{c}$ ' and ' $\mathrm{f}$ '. For that purpose, Eq. (51) can be integrated first, which results in Eq. (53) by swapping integration with summation. For computing the integral in Eq. (53), the lemma of Eq. (13) is applied. As a result, this lemma removes all sin/sin terms with different frequencies $j \omega t, k \omega t$, and only frequencies with $\mathrm{j}=\mathrm{k}=\mathrm{n}$ remain.

$$
\int_{0}^{T} c d t=\sum_{k=1}^{N} \sum_{j=1}^{N} \int_{0}^{T} \hat{u}_{j} \sin \left(j \omega t+\alpha_{j}\right) \hat{i}_{k} \sin \left(k \omega t+\beta_{k}\right) d t
$$

Thus, the integration of Eq. (53) results in Eq. (54) by additionally applying Eq. (12).

$$
\int_{0}^{T} c d t=\frac{T}{2} \sum_{n=1}^{N} \hat{u}_{n} \hat{i}_{n}\left(\cos \alpha_{n} \cos \beta_{n}+\sin \alpha_{n} \sin \beta_{n}\right)=\frac{T}{2} \sum_{n=1}^{N} \hat{u}_{n} \hat{i}_{n} \cos \left(\alpha_{n}-\beta_{n}\right)
$$

Furthermore, the integration of Eq. (52) is easy, because it is identical to Eq. (23), and the result can be taken from there, yielding Eq. (55).

$$
\int_{0}^{T} f d t=-R_{1} \frac{T}{2} \cdot \sum_{n=1}^{N} \hat{i}_{n}^{2}
$$

Out of Eq. (2), Eq. (54) and Eq. (55), we obtain the final result for the active power P according to Eq. (56).

$$
P=\frac{1}{2}\left\{\sum_{n=1}^{N} \hat{u}_{n} \hat{i}_{n} \cos \left(\alpha_{n}-\beta_{n}\right)-R_{1} \sum_{n=1}^{N} \hat{i}_{n}^{2}\right\}
$$

The result can be approximated for small $\mathrm{R}_{1}$ to Eq. (57).

$$
P \approx \frac{1}{2} \sum_{n=1}^{N} \hat{u}_{n} \hat{i}_{n} \cos \left(\alpha_{n}-\beta_{n}\right)
$$

\subsection{Apparent power $S$}

The apparent power S must be computed via Eq. (3) and Eq. (5). Again, Eq. (20) is used as starting point. The computation of $\mathrm{I}_{\text {eff }}$ is performed first, which translates into Eq. (59).

$$
\int_{0}^{T} i^{2}(t) d t=\int_{0}^{T}\left\{\sum_{n=1}^{N} \hat{i}_{n} \sin \left(n \omega t+\beta_{n}\right)\right\}^{2} d t
$$

The integral in this equation is identical to Eq. (31), yielding the same result as Eq. (33) in Eq. (59).

$$
I_{e f f}=\frac{1}{\sqrt{2}} \sqrt{\sum_{n=1}^{N} \hat{i}_{n}^{2}}
$$

Now, $\mathrm{U}_{\text {eff }}$ is calculated. It requires in the first step the squaring of $\mathrm{u}(\mathrm{t})$ according to Eq. (60).

$$
u^{2}(t)=\left[u_{1}(t)-i(t) R_{l}\right]^{2}=u_{1}^{2}-2 u_{1} i R_{1}+R_{l}^{2} i^{2}
$$

Subsequently, all three terms $\mathrm{u}_{l}{ }^{2},-2 \mathrm{u}_{l} \mathrm{i} \mathrm{R}_{l}$ and $\mathrm{R}_{l}{ }^{2} \mathrm{i}^{2}$ must be integrated. The integration of the first term is given by Eq. (61). This equation is structurally equivalent to Eq. (58). Thus, the result is Eq. (62). 


$$
\begin{aligned}
& \int_{0}^{T} u_{l}^{2}(t) d t=\int_{0}^{T}\left\{\sum_{n=1}^{N} \hat{u}_{n} \sin \left(n \omega t+\alpha_{n}\right)\right\}^{2} d t \\
& \int_{0}^{T} u_{l}^{2}(t) d t=\frac{T}{2} \sum_{n=1}^{N} \hat{u}_{n}^{2}
\end{aligned}
$$

The integration of the middle term is given by Eq. (63). This equation is structurally equivalent to Eq. (51)

$$
-2 R_{I} \int_{0}^{T} u_{l} i d t=-2 R_{I} \int_{0}^{T}\left\{\left(\sum_{j=1}^{N} \hat{u}_{j} \sin \left(j \omega t+\alpha_{j}\right)\right) \cdot \sum_{k=1}^{N} \hat{i}_{k} \sin \left(k \omega t+\beta_{k}\right)\right\} d t
$$

and Eq. (54). Thus, the integration of Eq. (63) results in Eq. (64).

$$
-2 R_{1} \int_{0}^{T} u_{1} i d t=-R_{1} T \sum_{n=1}^{N} \hat{u}_{n} \hat{i}_{n}\left(\cos \left(\alpha_{n}-\beta_{n}\right)\right)
$$

The integration of the last term can be obtained via Eq. (32) and results in Eq. (65).

$$
\int_{0}^{T} R_{l}^{2} i^{2}(t) d t=\frac{T}{2} R_{l}^{2} \sum_{k=1}^{N} \hat{i}_{k}^{2}
$$

Out of Eq. (62), Eq. (64) and Eq. (65), $\mathrm{U}_{\text {eff }}$ becomes Eq. (66).

$$
U_{\text {eff }}=\frac{1}{\sqrt{2}} \sqrt{\sum_{n=1}^{N} \hat{u}_{n}^{2}-2 R_{1} \sum_{n=1}^{N} \hat{u}_{n} \hat{i}_{n} \cos \left(\alpha_{n}-\beta_{n}\right)+R_{l}^{2} \sum_{n=1}^{N} \hat{i}_{n}^{2}}
$$

From Eq. (59) and Eq. (66), the final result for $\mathrm{S}$ is Eq. (67). For small $\mathrm{R}_{l}$, Eq. (68) holds approximately.

$$
\begin{aligned}
& S=\frac{1}{2} \sqrt{\sum_{n=1}^{N} \hat{u}_{n}^{2}-2 R_{1} \sum_{n=1}^{N} \hat{u}_{n} \hat{i}_{n} \cos \left(\alpha_{n}-\beta_{n}\right)+R_{l}^{2} \sum_{n=1}^{N} \hat{i}_{n}^{2}} \cdot \sqrt{\sum_{n=1}^{N} \hat{i}_{n}^{2}} \\
& S \approx \frac{1}{2} \sqrt{\sum_{n=1}^{N} \hat{u}_{n}^{2} \cdot \sum_{n=1}^{N} \hat{i}_{n}^{2}}
\end{aligned}
$$

\subsection{Reactive power $Q$}

According to the definition given in Eq. (4), the reactive power Q is computed out of Eq. (56) for P and out of Eq. (67) for S. Furthermore, P2 can be expanded to Eq. (69), and S2 can be expanded to Eq. (70).

$$
P^{2}=\frac{1}{4}\left[\begin{array}{c}
{\left[\sum_{n=1}^{N} \hat{u}_{n} \hat{i}_{n} \cos \left(\alpha_{n}-\beta_{n}\right)\right]^{2}} \\
-2 R_{1}\left[\sum_{n=1}^{N} \hat{u}_{n} \hat{i}_{n} \cos \left(\alpha_{n}-\beta_{n}\right)\right] \sum_{n=1}^{N} \hat{i}_{n}^{2} \\
R_{l}^{2}\left[\sum_{n=1}^{N} \hat{i}_{n}^{2}\right]^{2}
\end{array}\right]
$$




$$
S^{2}=\frac{1}{4}\left\{\left(\sum_{n=1}^{N} \hat{u}_{n}^{2}\right)\left(\sum_{n=1}^{N} \hat{i}_{n}^{2}\right)-2 R_{l}\left(\sum_{n=1}^{N} \hat{u}_{n} \hat{i}_{n} \cos \left(\alpha_{n}-\beta_{n}\right)\right)\left(\sum_{n=1}^{N} \hat{i}_{n}^{2}\right)+R_{l}^{2}\left(\sum_{n=1}^{N} \hat{i}_{n}^{2}\right)^{2}\right\}
$$

The subtraction of Eq. (70) from Eq. (69) delivers Eq. (71). Out of Eq. (71), we can compute Q as Eq. (73).

$$
\begin{aligned}
& Q^{2}=\frac{1}{4}\left\{\left[\sum_{n=1}^{N} \hat{u}_{n} \hat{i}_{n} \cos \left(\alpha_{n}-\beta_{n}\right)\right]^{2}-\left(\sum_{n=1}^{N} \hat{u}_{n}^{2}\right)\left(\sum_{n=1}^{N} \hat{i}_{n}^{2}\right)\right\} \\
& Q=\frac{1}{2} \sqrt{\left[\sum_{n=1}^{N} \hat{u}_{n} \hat{i}_{n} \cos \left(\alpha_{n}-\beta_{n}\right)\right]^{2}-\left(\sum_{n=1}^{N} \hat{u}_{n}^{2}\right)\left(\sum_{n=1}^{N} \hat{i}_{n}^{2}\right)}
\end{aligned}
$$

Interestingly, $\mathrm{Q}$ does not depend on $\mathrm{R}_{l}$. An approximation is thus not needed.

\subsection{Power factor $\lambda$}

The power factor $\lambda$ is computed out of Eq. (56) and Eq. (67), yielding Eq. (73).

$$
\lambda=\frac{\sqrt{\sum_{n=1}^{N} \hat{u}_{n} \hat{i}_{n} \cos \left(\alpha_{n}-\beta_{n}\right)-R_{l} \sum_{n=1}^{N} \hat{i}_{n}^{2}}}{\sqrt{\sum_{n=1}^{N} \hat{u}_{n}^{2}-2 R_{l} \sum_{n=1}^{N} \hat{u}_{n} \hat{i}_{n} \cos \left(\alpha_{n}-\beta_{n}\right)+R_{l}^{2} \sum_{n=1}^{N} \hat{i}_{n}^{2} \sqrt{\sum_{n=1}^{N} \hat{i}_{n}^{2}}}}
$$

This reduces to Eq. (74) for small $\mathrm{R}_{l}$.

$$
\lambda \approx \sqrt{\frac{\sum_{n=1}^{N} \hat{u}_{n} \hat{i}_{n} \cos \left(\alpha_{n}-\beta_{n}\right)}{\left(\sum_{n=1}^{N} \hat{u}_{n}^{2}\right)\left(\sum_{n=1}^{N} \hat{i}_{n}^{2}\right)}}
$$

\subsection{THDF for voltage and current at load $R$}

First, the THDF for the voltage $\mathrm{u}(\mathrm{t})$ at load $\mathrm{R}$ is computed according to its definition in Eq. (6) and by means of Eq. (66), yielding Eq. (75).

$$
T H D F_{u}=\frac{\sqrt{\sum_{n=2}^{N} \hat{u}_{n}^{2}-2 R_{I} \sum_{n=2}^{N} \hat{u}_{n} \hat{i}_{n} \cos \left(\alpha_{n}-\beta_{n}\right)+R_{l}^{2} \cdot \sum_{n=2}^{N} \hat{i}_{n}^{2}}}{\sqrt{\hat{u}_{l}^{2}-2 R_{l} \hat{u}_{l} \hat{i}_{l} \cos \left(\alpha_{1}-\beta_{l}\right)+R_{l}^{2} \cdot \hat{i}_{l}^{2}}}
$$

For small $\mathrm{R}_{l}$, this reduces to Eq. (76).

$$
\operatorname{THDF}_{u} \approx \frac{\sqrt{\sum_{n=2}^{N} \hat{u}_{n}^{2}}}{\hat{u}_{1}}
$$

The computation of the THDF for the current i(t) can be performed via Eq. (33), resulting in Eq. (77), which is the same result as in state 2 (Eq. (48)). Also Eq. (77) does not depend on $\mathrm{R}_{1}$. 


$$
\operatorname{THDF}_{i}=\frac{\sqrt{\sum_{n=2}^{N} \hat{i}_{n}^{2}}}{\hat{i}_{1}}
$$

\section{Results for State 4 (nonlin. gen. + nonlin. loads)}

For this state, the Thevenin equivalent voltage $\mathrm{u}_{l}(\mathrm{t})$ is modeled as a Fourier sum as given in Eq. (19). This sum is identical to the Fourier sum of state 3, i.e. to Eq. (18). The difference to state 3 is that every harmonic of $\mathrm{u}_{l}(\mathrm{t})$ creates a harmonic oscillation in $\mathrm{i}(\mathrm{t})$ that is additionally modulated by $\mathrm{R}$, resulting in more harmonic oscillations in $i(t)$ than in case 3. Because of that, the upper limit for the Fourier sum of $i(t)$ increases to $\mathrm{M}>\mathrm{N}$. However, since $\hat{\mathrm{u}}_{n}=0$ for $\mathrm{n}>\mathrm{N}$ holds, not many differences arise between state 3 and state 4 . Basically, only the summation of $\hat{\mathbf{1}}_{m}$ has to be executed until $\mathrm{M}$, while everything else remains the same. Because of that, the formulas of state 4 are very similar to those of state 3 . Therefore, no explicit derivation for them is given.

\subsection{Active power $P$}

We obtain the final result for the active power P according to Eq. (78).

$$
P=\frac{1}{2}\left\{\sum_{n=1}^{N} \hat{u}_{n} \hat{i}_{n} \cos \left(\alpha_{n}-\beta_{n}\right)-R_{1} \sum_{n=1}^{M} \hat{i}_{n}^{2}\right\}
$$

The result can be approximated for small $\mathrm{R}_{1}$ to Eq. (79).

$$
P \approx \frac{1}{2} \sum_{n=1}^{N} \hat{u}_{n} \hat{i}_{n}\left(\cos \left(\alpha_{n}-\beta_{n}\right)\right)
$$

\subsection{Apparent power $S$}

The final result for $\mathrm{S}$ is Eq. (80). For small $\mathrm{R}_{l}$, Eq. (81) holds approximately.

$$
\begin{aligned}
S & =\frac{1}{2} \sqrt{\sum_{n=1}^{N} \hat{u}_{n}^{2}-2 R_{I} \sum_{n=1}^{N} \hat{u}_{n} \hat{i}_{n} \cos \left(\alpha_{n}-\beta_{n}\right)+R_{l}^{2} \cdot \sum_{n=1}^{M} \hat{i}_{n}^{2}} \cdot \sqrt{\sum_{n=1}^{M} \hat{i}_{n}^{2}} \\
S & \approx \frac{1}{2} \sqrt{\sum_{n=1}^{N} \hat{u}_{n}^{2} \cdot \sum_{n=1}^{M} \hat{i}_{n}^{2}}
\end{aligned}
$$

\subsection{Reactive power $Q$}

We can compute Q as Eq. (82). Q does not depend on R1. An approximation ist thus not needed.

$$
Q=\frac{1}{2} \sqrt{\left[\sum_{n=1}^{N} \hat{u}_{n} \hat{i}_{n} \cos \left(\alpha_{n}-\beta_{n}\right)\right]^{2}-\left(\sum_{n=1}^{N} \hat{u}_{n}^{2}\right)\left(\sum_{n=1}^{M} \hat{i}_{n}^{2}\right)}
$$

\subsection{Power factor $\lambda$}

The power factor $\lambda$ is given in Eq. (83). This reduces to Eq. (84) for small $\mathrm{R}_{I}$.

$$
\lambda=\frac{\sqrt{\sum_{n=1}^{N} \hat{u}_{n} \hat{i}_{n} \cos \left(\alpha_{n}-\beta_{n}\right)-R_{I} \sum_{n=1}^{M} \hat{i}_{n}^{2}}}{\sqrt{\sum_{n=1}^{N} \hat{u}_{n}^{2}-2 R_{l} \sum_{n=1}^{N} \hat{u}_{n} \hat{i}_{n} \cos \left(\alpha_{n}-\beta_{n}\right)+R_{I}^{2} \sum_{n=1}^{M} \hat{i}_{n}^{2}} \cdot \sqrt{\sum_{n=1}^{M} \hat{i}_{n}^{2}}}
$$




$$
\lambda \approx \sqrt{\frac{\sum_{n=1}^{N} \hat{u}_{n} \hat{i}_{n} \cos \left(\alpha_{n}-\beta_{n}\right)}{\left(\sum_{n=1}^{N} \hat{u}_{n}^{2}\right)\left(\sum_{n=1}^{M} \hat{i}_{n}^{2}\right)}}
$$

\subsection{THDF for voltage and current at load $R$}

The THDF for the voltage $\mathrm{u}(\mathrm{t})$ at load $\mathrm{R}$ is as in Eq. (85). For small $\mathrm{R}_{l}$, this reduces to Eq. (86).

$$
\begin{aligned}
T H D F_{u} & =\frac{\sqrt{\sum_{n=2}^{N} \hat{u}_{n}^{2}-2 R_{l} \sum_{n=2}^{N} \hat{u}_{n} \hat{i}_{n} \cos \left(\alpha_{n}-\beta_{n}\right)+R_{l}^{2} \sum_{n=2}^{M} \hat{i}_{n}^{2}}}{\sqrt{\hat{u}_{1}^{2}-2 R_{1} \hat{u}_{1} \hat{i}_{1} \cos \left(\alpha_{1}-\beta_{1}\right)+R_{l}^{2} \cdot \hat{i}_{l}^{2}}} \\
\operatorname{THDF}_{u} \approx & \frac{\sqrt{\sum_{n=2}^{N} \hat{u}_{n}^{2}}}{\hat{u}_{1}}
\end{aligned}
$$

The formula of the THDF for the current i results in Eq. (87). Both, Eq. (86) and Eq. (87) do not depend on $\mathrm{R}_{l}$.

$$
T H D F_{i}=\frac{\sqrt{\sum_{n=2}^{M} \hat{i}_{n}^{2}}}{\hat{i}_{1}}
$$

\section{Conclusion}

New mathematical formulas for the active power $\mathrm{P}$, the apparent power $\mathrm{S}$, and the reactive power $\mathrm{Q}$ are given, together with equations for the power factor $\lambda$ and the distortion factors $\mathrm{THDF}_{u}$ and $\mathrm{THDF}_{i}$. These formulas are applicable for all combinations of linear/nonlinear generators and loads. The results are obtained by means of the Thevenin equivalent voltage $\mathrm{u}_{I}(\mathrm{t})$ of the power grid and its resistance $\mathrm{R}_{I}$ for every load R, and by modeling nonlinear generators and loads as finite Fourier sums. Furthermore, a method is given to obtain the coefficients of these Fourier sums by measurements in the power grid. The method's first advantage is that the load R can be of any kind, including phase-shifting, because no found formula depends on $\mathrm{R}$. The second advantage is that all formulas are given as exact and approximate solution for better handling. The approximations do not even depend on $\mathrm{R}_{l}$, which means that resistance measurements in the power grid become obsolete. Applications for the formulas are seen in photovoltaic energy systems and in industry 4.0, as well as in general power systems, because nonlinear generators and loads will prevail. In these use cases, the parameters $\mathrm{Q}, \lambda$ and THDF can be measured and computed as proposed and sent to the grid operator, who initiates a change in the filter settings of all stake holders of the power system to improve the quality of voltage and current.

\section{References}

[1] Croft T, Summers WI. American Electricians' Handbook. 11 ed., New York: McGraw Hill; 1987.

[2] Fink DG, Beaty HW. Standard Handbook for Electrical Engineers. 11 ed. New York: McGraw Hill; 1978.

[3] Dorf RC, Svoboda JA. Introduction to Electric Circuits. Chapter 5-Circuit Theorems. 8th ed. Hoboken, NJ: John Wiley \& Sons; 2010:162-207.

[4] IEEE. Trial-Use Standard Definitions for the Measurement of Electric Power Quantities under Sinusoidal, Nonsinusoidal, Balanced, or Unbalanced Conditions. IEEE Std. 1459-2000(2000).

[5] IEEE 10. The Authoritative Dictionary of IEEE Standards Terms. 7th ed. 
[6] Bracewell RN. The Fourier Transform and Its Applications. 3rd ed. Boston: McGraw-Hill; 2000.

[7] Katznelson Y. An Introduction to Harmonic Analysis. 2nd corrected ed. New York: Dover Publications; 1976.

[8] Trigonometry/A Square Wave in
http://en.wikibooks.org/wiki/Trigonometry/A_Square_Wave_in_Sines

Sines. [Online].

Available:

[9] Abramowitz M, Stegun IA. Handbook of Mathematical Functions with Formulas, Graphs, and Mathematical Tables. New York: Dover Publications; 1972.

[10] Hazewinkel M. Encyclopedia of Mathematics. Springer; 2001. 